TRANSACTIONS OF THE

AMERICAN MATHEMATICAL SOCIETY

Volume 362, Number 2, February 2010, Pages 603-621

S 0002-9947(09)04525-5

Article electronically published on September 14, 2009

\title{
MODULAR ACTIONS AND AMENABLE REPRESENTATIONS
}

\author{
INESSA EPSTEIN AND TODOR TSANKOV
}

\begin{abstract}
Consider a measure-preserving action $\Gamma \curvearrowright(X, \mu)$ of a countable group $\Gamma$ and a measurable cocycle $\alpha: X \times \Gamma \rightarrow \operatorname{Aut}(Y)$ with countable image, where $(X, \mu)$ is a standard Lebesgue space and $(Y, \nu)$ is any probability space. We prove that if the Koopman representation associated to the action $\Gamma \curvearrowright X$ is non-amenable, then there does not exist a countable-to-one Borel homomorphism from the orbit equivalence relation of the skew product action $\Gamma \curvearrowright^{\alpha} X \times Y$ to the orbit equivalence relation of any modular action (i.e., an inverse limit of actions on countable sets or, equivalently, an action on the boundary of a countably-splitting tree), generalizing previous results of Hjorth and Kechris. As an application, for certain groups, we connect antimodularity to mixing conditions. We also show that any countable, non-amenable, residually finite group induces at least three mutually orbit inequivalent free, measure-preserving, ergodic actions as well as two non-Borel bireducible ones.
\end{abstract}

\section{INTRODUCTION}

Let $\Gamma$ be a countable, infinite group which acts in a Borel way on a standard Borel space $X$. The action gives rise to a Borel orbit equivalence relation $E_{\Gamma}^{X}$ with countable classes. Conversely, every countable Borel equivalence relation is given by a group action (Feldman-Moore [7). It is of interest to compare equivalence relations arising from different groups and different actions of the same group.

Let $E, F$ be equivalence relations on the spaces $X, Y$, respectively. A homomorphism from $E$ to $F$ is a map $f: X \rightarrow Y$ such that

$$
x E y \Longrightarrow f(x) F f(y)
$$

for all $x, y \in X$. A map is countable-to-one if the preimage of every point is countable. Countable-to-one homomorphisms occur in different contexts: examples arise from orbit equivalences and stable orbit equivalences as well as Borel reductions between countable equivalence relations. A countable-to-one homomorphism is, in fact, a combination of an inclusion and a Borel reduction (see Thomas 24, Section 4]). In the Borel setting, one is interested in Borel homomorphisms, while in the presence of a measure, one usually considers measurable homomorphisms which are defined only almost everywhere.

Following Hjorth 13], call a Borel group action on a standard Borel space $X$ modular if there exists a sequence of countable Borel partitions $\mathcal{A}_{1} \succ \mathcal{A}_{2} \succ \cdots$

Received by the editors March 22, 2007 and, in revised form, April 12, 2007.

2000 Mathematics Subject Classification. Primary 37A20; Secondary 22D10.

Key words and phrases. Modular actions, amenable representations, orbit equivalence, Borel reducibility.

The first author's research was partially supported by NSF grant 443948-HJ-21632.

The second author's research was partially supported by NSF grant and DMS-0455285. 
of $X$, each one refining the previous, which separate points in $X$ and are invariant under the action. Note that if there is a $\Gamma$-invariant, ergodic measure on $X$, then, possibly excluding a null set, all partitions are finite and the action on each partition is transitive. It is shown in 21] that, on an invariant set of full measure, every measure-preserving, ergodic, modular action is isomorphic to an action on the boundary of a rooted, locally finite tree induced by an action by automorphisms on the tree or, which is the same, an inverse limit of actions on finite sets. Also, it is not hard to see that a group admits a free, ergodic, modular action iff it is residually finite (i.e., the intersection of all of its normal subgroups of finite index is trivial); see 21] again.

Say that a countable Borel equivalence relation is of modular type if it is induced by a modular action. Hjorth considered equivalence relations of modular type in order to show that there exist more than two treeable equivalence relations (up to Borel bireducibility), which was an important problem in the theory of Borel equivalence relations. (An equivalence relation is treeable if to each equivalence class can be assigned in a Borel way the structure of a tree.) More precisely, he proved the following result:

Theorem 1.1 (Hjorth [13). Let $\Gamma \curvearrowright X$ be a modular action and $E$ be the orbit equivalence relation. Let $M \subseteq 2^{\mathbf{F}_{2}}$ be a set of full measure (where $\mathbf{F}_{2}$ is the free group with 2 generators and $2^{\mathbf{F}_{2}}$ is equipped with the standard Bernoulli measure and the shift action of $\mathbf{F}_{2}$ ). Then there does not exist a countable-to-one Borel homomorphism from $\left.E_{\mathbf{F}_{2}}^{2^{\mathbf{F}_{2}}}\right|_{M}$ to $E$.

The above theorem implies that any free, measure-preserving, modular action of $\mathbf{F}_{2}$ gives rise to an intermediate treeable equivalence relation. (For more on the theory of countable Borel equivalence relations and in particular the treeable ones, see Jackson-Kechris-Louveau [15].) It is interesting to try to generalize Theorem 1.1 to include actions other than $\mathbf{F}_{2} \curvearrowright 2^{\mathbf{F}_{2}}$. Kechris 21] defined the notion of an antimodular action (one whose orbit equivalence relation does not admit a countable-to-one homomorphism to an equivalence relation of modular type) and, in the presence of an invariant measure, isolated a representation-theoretic property which implies antimodularity. Since in most of our considerations below we will have a measure present, we find it convenient to introduce a notion of a.e. antimodularity: we say that a measure-preserving action $\Gamma \curvearrowright(X, \mu)$ is $\mu$-antimodular if its restriction to any invariant conull subset of $X$ is antimodular (or, equivalently, for any (not necessarily invariant) conull $A \subseteq X$, the restricted equivalence relation $\left.E_{\Gamma}^{X}\right|_{A}$ does not admit a countable-to-one homomorphism to an equivalence relation of modular type).

Recall that if $\Gamma$ acts on $X$ preserving a measure $\mu$, the Koopman representation $\kappa$ of $\Gamma$ on the Hilbert space $L^{2}(X, \mu)$ is the unitary representation given by

$$
(\kappa(\gamma) f)(x)=f\left(\gamma^{-1} \cdot x\right)
$$

We will usually consider the restriction $\kappa_{0}$ of $\kappa$ to the orthogonal complement of the constant functions $L_{0}^{2}(X)=\left\{f \in L^{2}(X) \mid \int f=0\right\}$ and, by abuse of terminology, also call it the Koopman representation. If $\sigma$ and $\pi$ are unitary representations of the same group, we write $\sigma \leq \pi$ if $\sigma$ is contained in $\pi$ (i.e., is isomorphic to a subrepresentation of $\pi$ ) and $\sigma \prec \pi$ if $\sigma$ is weakly contained in $\pi$. For all necessary background on unitary representations, an excellent reference is Bekka-de la HarpeValette [1]. The action of $\Gamma$ on $X$ is called tempered if $\kappa_{0} \prec \lambda_{\Gamma}$, where $\lambda_{\Gamma}$ is the 
left-regular representation of $\Gamma$. Kechris [21] adapted Hjorth's method from [13] to show that if $\mathbf{F}_{2} \leq \Gamma$, then every tempered action of $\Gamma$ is antimodular. He also asked whether the hypotheses of this theorem can be weakened, for example, whether " $\mathbf{F}_{2} \leq \Gamma$ " can be replaced by " $\Gamma$ is non-amenable" and "the action is tempered" by " $\kappa_{0}$ does not weakly contain a finite-dimensional representation of $\Gamma$." (If $\Gamma$ is amenable, then by well-known results of Dye and Ornstein-Weiss (see [17]), the orbit equivalence relation is hyperfinite, and therefore induced by a modular action of $\mathbb{Z}$, on a set of measure 1.) In the present paper we answer those two questions, the first one in the affirmative and the second in the negative (cf. Corollary 1.4 and Proposition 5.21).

It turns out that another representation-theoretic property of measure-preserving actions is relevant in this situation, namely the property of $\kappa_{0}$ being amenable in the sense of Bekka [3. We recall the definition and a few basic facts from [3. A unitary representation $\pi$ of $\Gamma$ on a Hilbert space $\mathcal{H}$ is amenable if there exists a $\Gamma$-invariant state on the $\mathrm{C}^{*}$-algebra $B(\mathcal{H})$ of bounded linear operators on $\mathcal{H}$, i.e., a bounded linear functional $M$ on $B(\mathcal{H})$ satisfying $M \geq 0, M(I)=1$, and

$$
M\left(\pi(\gamma) S \pi\left(\gamma^{-1}\right)\right)=M(S)
$$

for all $\gamma \in \Gamma$ and $S \in B(\mathcal{H})$. The notion of an amenable representation captures many known instances of amenability in a single framework. For example, a group is amenable iff all of its representations are amenable, an action of a countable group on a countable set $I$ is amenable iff the corresponding representation on $\ell^{2}(I)$ is amenable (cf. Lemma 3.1 and the remark after it), etc. A useful characterization of amenability is the following:

$$
\pi \text { is amenable } \Longleftrightarrow 1_{\Gamma} \prec \pi \otimes \bar{\pi}
$$

2, Theorem 5.1]. The latter condition is sometimes referred to as the absence of stable spectral gap. For more examples and further discussion, see [3]. (In [3], the theory is developed for locally compact groups, but we only need the discrete case here.)

Let $(X, \mu)$ be a standard Lebesgue space (i.e., a standard Borel space equipped with a non-atomic, Borel, probability measure $\mu$ ) and $\Gamma$ a countable group acting by measure-preserving transformations on it. Now consider another (arbitrary) probability space $(Y, \nu)$ and a measurable cocycle $\alpha: X \times \Gamma \rightarrow \operatorname{Aut}(Y)$, where $\operatorname{Aut}(Y)$ denotes the group of measure-preserving automorphisms of $Y$. $\alpha$ gives rise to a measure-preserving action $\Gamma \curvearrowright^{\alpha} X \times Y$ as follows:

$$
\gamma \cdot(x, y)=(\gamma \cdot x, \alpha(x, \gamma) \cdot y) \text {. }
$$

Conversely, by a well-known theorem of Rokhlin, every ergodic extension of the action $\Gamma \curvearrowright X$ arises in this fashion (see [11, 3.3]).

Now we can state the main theorem of this paper.

Theorem 1.2. Let $\Gamma$ act by measure-preserving transformations on the standard Lebesgue space $(X, \mu)$. Let $(Y, \nu)$ be an arbitrary probability space and $\alpha: X \times \Gamma \rightarrow$ $\operatorname{Aut}(Y)$ a measurable cocycle whose image is contained in a countable subgroup of $\operatorname{Aut}(Y)$. Then, if the Koopman representation $\kappa_{0}$ associated with the action $\Gamma \curvearrowright X$ is not amenable, the action $\Gamma \curvearrowright^{\alpha} X \times Y$ is $\mu \times \nu$-antimodular.

Remark. We do not know whether the condition that the image of $\alpha$ is countable is necessary. 
Note the following immediate corollary which is obtained in the case when $Y$ consists of a single point.

Corollary 1.3. Suppose that $\Gamma \curvearrowright(X, \mu)$ is measure-preserving. Then if $\kappa_{0}$ is non-amenable, the equivalence relation $E_{\Gamma}^{X}$ is $\mu$-antimodular.

We can apply that to a variety of situations where we know that the Koopman representation is non-amenable and produce examples of antimodular actions. For example, if $\Gamma$ is non-amenable, then its left-regular representation is not amenable [3, Theorem 2.2] and

$$
\rho \text { is non-amenable and } \pi \prec \rho \Longrightarrow \pi \text { is non-amenable }
$$

3. Corollary 5.3], so we have:

Corollary 1.4. Every tempered action of a non-amenable group is antimodular.

Recall that an action $\Gamma \curvearrowright(X, \mu)$ is called weakly mixing if the Koopman representation $\kappa_{0}$ does not contain finite-dimensional subrepresentations and mixing if $\kappa_{0}$ is a $c_{0}$-representation, i.e.,

$$
\left\langle\kappa_{0}(\gamma) f, f\right\rangle \rightarrow 0 \quad \text { as } \quad \gamma \rightarrow \infty
$$

for all $f \in L_{0}^{2}(X)$. It is clear that a weakly mixing action cannot be modular and Kechris 21] asked whether weak mixing (or even mixing) always implies antimodularity. One has to exclude amenable groups from consideration, however, since any ergodic action of an amenable group is orbit equivalent to a modular action of $\mathbb{Z}$. We will see in Section 5 that in general weak mixing (and even the stronger condition that $\kappa_{0}$ does not weakly contain a finite-dimensional representation) does not imply antimodularity. However, such an implication does exist for certain groups and for special actions of arbitrary non-amenable groups as we see below.

If a group has property $(\mathrm{T})$, then all of its amenable representations contain a finite-dimensional subrepresentation (the converse is also true; cf. Bekka-Valette 2]) and hence:

Corollary 1.5. Let $\Gamma$ have property $(T)$. Then every weakly mixing $\Gamma \curvearrowright(X, \mu)$ is $\mu$-antimodular.

Recall that a group $\Gamma$ has the Haagerup approximation property (HAP) if it has a $c_{0}$-representation $\pi$ such that $1_{\Gamma} \prec \pi$. (For more on groups with HAP, see Cherix et al. [4.) Since for any representation $\pi$, if $\pi$ is a $c_{0}$-representation, then $\pi \otimes \pi$ is also a $c_{0}$-representation, using (1.1), we obtain:

Corollary 1.6. If $\Gamma$ does not have $H A P$, every mixing action $\Gamma \curvearrowright(X, \mu)$ is $\mu$ antimodular.

A class of actions for which mixing implies antimodularity for arbitrary nonamenable groups is given by the generalized Bernoulli shifts (cf. Corollary 3.4). We do not know an example of a mixing action of a non-amenable group which is not antimodular.

Our final application is to the theory of orbit equivalence and Borel reducibility. We use Theorem 1.2 to show that every residually finite, non-amenable group admits at least three non-orbit equivalent actions as well as two non-Borel bireducible ones. For general non-amenable groups, it is only known that they admit at least two non-orbit equivalent actions (Schmidt 22], Connes-Weiss [5], Hjorth [13]). For definitions and further discussion, see Section 4 . 
The organization of the paper is as follows. In Section 2, we prove Theorem 1.2 in Section 3, generalized Bernoulli shifts and actions on compact Polish groups by automorphisms are considered; in Section 4, we discuss the applications to orbit equivalence and Borel reducibility; and finally, in Section 5, we give an example which shows that the hypothesis in Corollary 1.3 cannot be replaced by the weaker " $\kappa_{0}$ does not weakly contain a finite-dimensional representation," answering the previously mentioned question of Kechris.

Below $\Gamma$ will always be a countable, infinite group and $Q \subseteq \Gamma$ a finite set. All vector spaces will be complex and all representations unitary.

\section{Proof of Theorem 1.2}

We argue towards a contradiction. Suppose that the action $\Gamma \curvearrowright X \times Y$ is not $\mu \times \nu$-antimodular. Suppose also that the image of $\alpha$ is contained in the countable subgroup $\Lambda$ of $\operatorname{Aut}(Y)$. Let $\operatorname{MALG}(X)$ denote the measure algebra of $(X, \mu)$. Let $Z=\mathbb{N} \times Y$ and let $\sigma$ be the measure on $Z$ which is the product of the counting measure on $\mathbb{N}$ and $\nu$.

The following technical proposition extracts from the combinatorial information given by the existence of a homomorphism to an equivalence relation of modular type the data we need to construct a $\kappa_{0}$-invariant state on $B\left(L_{0}^{2}(X)\right)$.

Proposition 2.1. For every finite $Q \subseteq \Gamma$ and $\epsilon>0$, there exists a Borel map $\Phi: Z \rightarrow \operatorname{MALG}(X)$ such that the following are satisfied:

(i) for almost all $x \in X$,

$$
\int_{Z} \chi_{\{x \in \Phi(z)\}}(z) \mathrm{d} \sigma(z)=1
$$

$$
\int_{\{\mu(\Phi(z))>\epsilon\}} \mu(\Phi(z)) \mathrm{d} \sigma(z)<\epsilon
$$

(iii) for all $\tau \in Q$, there exists $T \in \operatorname{Aut}(Z, \sigma)$ such that

$$
\int_{Z} \mu(\Phi(T z) \triangle \tau \cdot \Phi(z)) \mathrm{d} \sigma(z)<\epsilon .
$$

To visualize what the proposition claims, it helps to consider the case when $Y$ is a single point. Then condition (ii) says that $\Phi$ defines a partition of $X$ into countably many pieces, condition (iii) says that all pieces have measure smaller than $\epsilon$, and, finally, condition (iii) says that the partition is "almost invariant" with respect to the pair $(Q, \epsilon)$.

Proof. Let $\Delta$ be a countable group which acts modularly on a standard Borel space $W$. We suppose that there is a conull $C \subseteq X \times Y$ and a countable-to-one Borel homomorphism $\theta: C \rightarrow W$ from $E_{\Gamma}^{C}$ to $E_{\Delta}^{W}$. By [21, 1.2], we can assume that $\theta$ is injective. Fix a symmetric $Q \subseteq \Gamma$ containing 1 and $1 / 2>\epsilon>0$. Denote by $\mathcal{B}$ the Borel $\sigma$-algebra of $W$ and let

$$
\mathcal{B}_{0} \subseteq \mathcal{B}_{1} \subseteq \cdots \subseteq \mathcal{B}_{k} \subseteq \mathcal{B}_{k+1} \subseteq \cdots \subseteq \mathcal{B}
$$

be atomic (finite or countable) Boolean algebras which witness that the action $\Delta \curvearrowright W$ is modular, i.e., each $\mathcal{B}_{k}$ is invariant under $\Delta$ and $\bigcup_{k} \mathcal{B}_{k}$ generates $\mathcal{B}$. If $B \in \mathcal{B}$ and $A \subseteq X \times Y$, denote

$$
\hat{B}=\theta^{-1}(B) \quad \text { and } \quad A^{y}=\{x \in X \mid(x, y) \in A\} .
$$


Since $\theta$ is a Borel homomorphism, there exists a Borel map $g: C \times \Gamma \rightarrow \Delta$ such that

$$
g((x, y), \tau) \cdot \theta(x, y)=\theta(\tau \cdot(x, y)) .
$$

Lemma 2.2. For any $\eta>0$, there is a Borel set $M \subseteq C$ and $k \in \mathbb{N}$ such that the following hold:

(1) $\mu \times \nu(M)>1-\eta$;

(2) for any $\tau \in Q$, the functions $(x, y) \mapsto g((x, y), \tau)$ and $(x, y) \mapsto \alpha(x, \tau)$ are constant on $\hat{B} \cap M$ for each atom $B \in \mathcal{B}_{k}$;

(3) if $(x, y) \in \hat{B} \cap M$ for some atom $B \in \mathcal{B}_{k}$, then $\mu\left(\hat{B}^{y}\right)<\eta$;

(4) the set $\left\{B \in \mathcal{B}_{k}: M \cap \hat{B} \neq \emptyset\right\}$ is finite.

The lemma and proof are similar to [13, Claim I]. However, we additionally require that the cocycle $\alpha$ be constant on the atoms and $M$ only intersect atoms with vertical sections of sufficiently small measure.

Proof. It suffices to find the required pair $(M, k)$ for a single element $\tau \in Q$. Indeed, since $Q$ is finite, in the end, we can take the intersection of the $M$ 's and the maximum of the $k$ 's.

Let $\Delta_{0} \subseteq \Delta, \Lambda_{0} \subseteq \Lambda$ be finite sets such that off a set of $\mu \times \nu$-measure less than $\eta / 2$, we have $g((x, y), \tau) \in \Delta_{0}$ and $\alpha(x, \tau) \in \Lambda_{0}$. Partition $X$ into $A_{1}, \ldots, A_{m} \subseteq X$ such that $\mu\left(A_{j}\right)<\eta / 2$. Then for $\delta \in \Delta_{0}, \lambda \in \Lambda_{0}$, and $j \leq m$, let

$$
M(\delta, \lambda, j)=\left\{(x, y) \in C \mid g((x, y), \tau)=\delta \text { and } \alpha(x, \tau)=\lambda \text { and } x \in A_{j}\right\} .
$$

We have

$$
\bigcup_{\substack{\delta \in \Delta_{0}, \lambda \in \Lambda_{0} \\ j \leq m}} M(\delta, \lambda, j) \geq 1-\eta / 2 .
$$

By the assumption that $\theta$ is injective, the set of $\theta$-preimages of $\bigcup_{k} \mathcal{B}_{k}$ is dense in the measure algebra of $X \times Y$. Thus, for any $\delta \in \Delta_{0}, \lambda \in \Lambda_{0}$, and $j \leq m$, there are $k(\delta, \lambda, j) \in \mathbb{N}$ and $B(\delta, \lambda, j) \in \mathcal{B}_{k(\delta, \lambda, j)}$ such that

$$
\mu \times \nu(\hat{B}(\delta, \lambda, j) \triangle M(\delta, \lambda, j))<\frac{\eta}{6 m\left|\Delta_{0}\right|\left|\Lambda_{0}\right|} .
$$

Also, since $M(\delta, \lambda, j) \subseteq A_{j}$ and $\mu\left(A_{j}\right) \leq \eta / 2$,

$$
\begin{aligned}
\int_{\left\{y \in Y \mid \mu\left(\hat{B}(\delta, \lambda, j)^{y}\right)>\eta\right\}} \mu\left(\hat{B}(\delta, \lambda, j)^{y}\right) \mathrm{d} \nu(y) & \leq 2 \int_{Y} \mu\left(\hat{B}(\delta, \lambda, j)^{y} \triangle M(\delta, \lambda, j)^{y}\right) \mathrm{d} \nu(y) \\
& =2 \mu \times \nu(\hat{B}(\delta, \lambda, j) \triangle M(\delta, \lambda, j)) \\
& <\frac{\eta}{3 m\left|\Delta_{0}\right|\left|\Lambda_{0}\right|}
\end{aligned}
$$

and hence,

$$
\mu\left(\bigcup_{\substack{\delta \in \Delta_{0}, \lambda \in \Lambda_{0} \\ j \leq m}}\left\{(x, y) \in \hat{B}(\delta, \lambda, j) \mid \mu\left(\hat{B}(\delta, \lambda, j)^{y}\right)>\eta\right\}\right)<\eta / 3 .
$$


Finally, let $k=\max \{k(\delta, \lambda, j)\}$ and

$$
\begin{aligned}
M= & \bigcup_{\substack{\delta \in \Delta_{0}, \lambda \in \Lambda_{0}, j \leq m}} \hat{B}(\delta, \lambda, j) \cap M(\delta, \lambda, j) \\
& \backslash \bigcup_{\substack{\delta \in \Delta_{0}, \lambda \in \Lambda_{0}, j \leq m}}\left\{(x, y) \in \hat{B}(\delta, \lambda, j) \mid \mu\left(\hat{B}(\delta, \lambda, j)^{y}\right)>\eta\right\} .
\end{aligned}
$$

Then (22), (3), and (41) are satisfied by definition, and by (2.1), (2.2), and (2.3),

$$
\mu \times \nu(M)>1-(\eta / 2+\eta / 6+\eta / 3)=1-\eta,
$$

which verifies (1).

Apply Lemma 2.2 with $\eta=\epsilon^{2} / 4$ to obtain $M$ and $k$ and fix them from now on. Let $\left\{B_{i} \mid i \in \mathbb{N}\right\}$ enumerate the atoms of $\mathcal{B}_{k}$ (if $\mathcal{B}_{k}$ contains only finitely many atoms, add empty sets to the enumeration). The functions $(x, y) \mapsto g((x, y), \tau)$ and $(x, y) \mapsto \alpha(x, \tau)$ are constant on each $\hat{B}_{i} \cap M$ and, abusing notation, we will write $g(i, \tau)$ and $\alpha(i, \tau)$ (for those $i$ for which $\hat{B}_{i} \cap M \neq \emptyset$ ). Now define the map $\Phi: Z \rightarrow \operatorname{MALG}(X)$ by $\Phi(i, y)=\hat{B}_{i}^{y}$. Note that $\left\{\hat{B}_{i}\right\}$ is a partition of $X \times Y$ and hence, for almost every $y \in Y,\{\Phi(i, y) \mid i \in \mathbb{N}\}$ is a partition of $X$.

That condition (ii) is satisfied follows from the fact that for each $x$, the collection $\{\{y \mid x \in \Phi(i, y)\} \mid i \in \mathbb{N}\}$ forms a partition of $Y$. We proceed to check (iii). Using Lemma 2.2 (11) and (3), we have:

$$
\begin{aligned}
\int_{\{\mu(\Phi(z))>\epsilon\}} \mu(\Phi(z)) \mathrm{d} \sigma(z) & =\int_{X \times Y \times \mathbb{N}} \chi_{\left\{(x, y) \in \hat{B}_{i} \text { and } \mu\left(\hat{B}_{i}^{y}\right)>\epsilon\right\}} \mathrm{d}(x, y, i) \\
& \leq \int_{((X \times Y) \backslash M) \times \mathbb{N}} \chi_{\left\{(x, y) \in \hat{B}_{i}\right\}} \mathrm{d}(x, y, i) \\
& =\mu((X \times Y) \backslash M)<\eta<\epsilon .
\end{aligned}
$$

We are left with verifying (iii). Fix $\tau \in Q$. We will construct $T \in \operatorname{Aut}(Z)$ such that

Set

$$
\int_{Z} \mu(\Phi(T z) \triangle \tau \cdot \Phi(z)) \mathrm{d} \sigma(z) \leq 7 \epsilon .
$$

We have

$$
G=\left\{(i, y) \in Z \mid \frac{\mu\left(\Phi(i, y) \backslash M^{y}\right)}{\mu(\Phi(i, y))}<\epsilon\right\} .
$$

$$
\begin{aligned}
\int_{Z \backslash G} \mu(\Phi(i, y)) \mathrm{d} \sigma(i, y) & \leq \frac{1}{\epsilon} \int_{Z \backslash G} \mu\left(\Phi(i, y) \backslash M^{y}\right) \mathrm{d} \sigma(i, y) \\
& \leq \frac{1}{\epsilon} \mu \times \nu(X \times Y \backslash M) \leq \epsilon / 4 .
\end{aligned}
$$

Define

$$
\begin{gathered}
N_{0}=\{(x, y) \in M \mid \exists i \quad(i, y) \in G \text { and } x \in \Phi(i, y)\}, \\
N=N_{0} \cap \tau^{-1} \cdot N_{0} .
\end{gathered}
$$

By (2.4),

and hence,

$$
\mu \times \nu\left(N_{0}\right) \geq 1-\mu \times \nu(C \backslash M)-\epsilon / 4 \geq 1-\epsilon / 2
$$

$$
\mu \times \nu(X \times Y \backslash N) \leq 2(\epsilon / 2)=\epsilon
$$


Let $Z_{0}=\left\{(i, y) \in Z \mid \Phi(i, y) \cap N^{y} \neq \emptyset\right\}$. Notice that

$$
\begin{aligned}
\int_{Z \backslash Z_{0}} \mu(\Phi(z)) \mathrm{d} \sigma(z) & =\int_{Y} \sum_{\left\{i \mid \Phi(i, y) \cap N^{y}=\emptyset\right\}} \mu(\Phi(i, y)) \mathrm{d} \nu(y) \\
& \leq \int_{Y} \mu\left(X \backslash N^{y}\right) \mathrm{d} \nu(y) \\
& \leq \mu \times \nu(X \times Y \backslash N) \leq \epsilon .
\end{aligned}
$$

Define the partial automorphism $T_{0}: Z_{0} \rightarrow Z$ by

$$
T_{0}(i, y)=(j, \alpha(i, \tau) \cdot y) \Longleftrightarrow g(i, \tau) \cdot B_{i}=B_{j} .
$$

Lemma 2.3. Given $(i, y)=z \in Z_{0}$, the following hold:

(1) $\tau \cdot\left(\Phi(i, y) \cap M^{y}\right) \subseteq \Phi\left(T_{0}(i, y)\right)$;

(2) $\tau^{-1} \cdot\left(\Phi\left(T_{0}(i, y)\right) \cap M^{\alpha(i, \tau) \cdot y}\right) \subseteq \Phi(i, y)$;

(3) $\frac{\mu(\Phi(z))}{\mu\left(\Phi\left(T_{0} z\right)\right)} \in\left[1-\epsilon, \frac{1}{1-\epsilon}\right] \subseteq(1-2 \epsilon, 1+2 \epsilon)$;

(4) $\mu\left(\tau \cdot \Phi(z) \triangle \Phi\left(T_{0} z\right)\right) \leq 3 \epsilon \cdot \mu\left(\Phi\left(T_{0} z\right)\right)$;

(5) $T_{0}$ is injective and measure preserving.

Proof. (11). Take $x \in \Phi(i, y) \cap M^{y}$. Then

$$
\theta(\tau \cdot(x, y))=g(i, \tau) \cdot \theta(x, y) \in g(i, \tau) \cdot B_{i}=B_{j}
$$

for some $j$. So $\tau \cdot(x, y) \in \hat{B}_{j}$. Also, $\tau \cdot(x, y)=(\tau \cdot x, \alpha(i, \tau) \cdot y)$. By our definition of $T_{0}, \tau \cdot(x, y) \in \Phi\left(T_{0}(i, y)\right)$.

(2). Let $x \in \Phi\left(T_{0}(i, y)\right) \cap M^{\alpha(i, \tau) \cdot y}$ and let $j$ be such that $T_{0}(i, y)=(j, \alpha(i, \tau) \cdot y)$. Then $(x, \alpha(i, \tau) \cdot y) \in \hat{B}_{j}$. Let $x_{1} \in \Phi(i, y) \cap N^{y}$. From (1),

$$
\tau \cdot x_{1} \in \Phi\left(T_{0}(i, y)\right) \cap M^{\alpha(i, \tau) \cdot y} .
$$

Note that

$$
\tau^{-1} \cdot\left(\tau \cdot\left(x_{1}, y\right)\right)=\left(x_{1}, y\right) \in \hat{B}_{i} .
$$

This implies that $g\left(j, \tau^{-1}\right) \cdot B_{j}=B_{i}$. So then

$$
\left(\tau^{-1} \cdot x, \alpha\left(x, \tau^{-1}\right) \alpha(i, \tau) \cdot y\right)=\tau^{-1} \cdot(x, \alpha(i, \tau) \cdot y) \in \hat{B}_{i} .
$$

Also, $\alpha(i, \tau)=\alpha\left(x_{1}, \tau\right)$ and $\alpha\left(j, \tau^{-1}\right)=\alpha\left(\tau \cdot x_{1}, \tau^{-1}\right)$. By the cocycle identity,

$$
\alpha\left(x, \tau^{-1}\right) \alpha(i, \tau)=\alpha\left(j, \tau^{-1}\right) \alpha(i, \tau)=\alpha\left(\tau \cdot x_{1}, \tau^{-1}\right) \alpha\left(x_{1}, \tau\right)=1,
$$

and hence, combining with (2.6),$\tau^{-1} \cdot x \in \Phi(i, y)$.

(3). From (11), we have that $\mu\left(M^{y} \cap \Phi(i, y)\right) \leq \mu\left(\Phi\left(T_{0}(i, y)\right)\right)$. Since $\Phi(i, y) \cap$ $N^{y} \neq \emptyset,(i, y) \in G$, and we obtain

$$
\mu\left(\Phi\left(T_{0}(i, y)\right)\right) \geq \mu\left(M^{y} \cap \Phi(i, y)\right) \geq(1-\epsilon) \mu(\Phi(i, y)),
$$

which then allows us to conclude that

$$
\frac{\mu(\Phi(i, y))}{\mu\left(\Phi\left(T_{0}(i, y)\right)\right)} \leq \frac{1}{1-\epsilon} .
$$

Similarly, by (2) and the fact that $T_{0}(i, y) \in G$,

$$
\mu(\Phi(i, y)) \geq \mu\left(\Phi\left(T_{0}(i, y)\right) \cap M^{\alpha(i, \tau) \cdot y}\right) \geq(1-\epsilon) \mu\left(\Phi\left(T_{0}(i, y)\right)\right),
$$

which then leads to

$$
\frac{\mu(\Phi(i, y))}{\mu\left(\Phi\left(T_{0}(i, y)\right)\right)} \geq 1-\epsilon
$$


(44). Using the fact that the action of $\Gamma$ on $X$ is measure-preserving and also using (11) and (3), we have

$$
\begin{aligned}
\mu\left(\tau \cdot \Phi(i, y) \backslash \Phi\left(T_{0}(i, y)\right)\right) & =\mu\left(\Phi(i, y) \backslash \tau^{-1} \cdot \Phi\left(T_{0}(i, y)\right)\right) \\
& \leq \mu\left(\Phi(i, y) \backslash M^{y}\right) \\
& <\epsilon \cdot \mu(\Phi(i, y)) \\
& <\epsilon(1+2 \epsilon) \mu\left(\Phi\left(T_{0}(i, y)\right)\right) \\
& <2 \epsilon \cdot \mu\left(\Phi\left(T_{0}(i, y)\right)\right) .
\end{aligned}
$$

Similarly, using (2), $\mu\left(\tau^{-1} \cdot \Phi\left(T_{0}(i, y)\right) \backslash \Phi(i, y)\right)<\epsilon \cdot \mu\left(\Phi\left(T_{0}(i, y)\right)\right)$.

(5). Suppose that $T_{0}\left(i_{1}, y_{1}\right)=T_{0}\left(i_{2}, y_{2}\right)$ for some $\left(i_{1}, y_{1}\right),\left(i_{2}, y_{2}\right) \in Z_{0}$. Take $x_{1} \in \Phi\left(i_{1}, y_{1}\right) \cap N^{y_{1}}, x_{2} \in \Phi\left(i_{2}, y_{2}\right) \cap N^{y_{2}}$. Let

$$
B_{j}=g\left(i_{1}, \tau\right) \cdot B_{i_{1}}=g\left(i_{2}, \tau\right) \cdot B_{i_{2}} .
$$

$\tau \cdot\left(x_{1}, y_{1}\right), \tau \cdot\left(x_{2}, y_{2}\right) \in \hat{B}_{j} \cap M$, so

$$
B_{i_{1}}=g\left(j, \tau^{-1}\right) \cdot B_{j}=B_{i_{2}} .
$$

Hence $i_{1}=i_{2}$ and

$$
y_{1}=\alpha\left(i_{1}, \tau\right)^{-1}\left(\alpha\left(i_{1}, \tau\right) \cdot y_{1}\right)=\alpha\left(i_{2}, \tau\right)^{-1}\left(\alpha\left(i_{2}, \tau\right) \cdot y_{2}\right)=y_{2} .
$$

Now let $A \subseteq Z_{0}$. We claim that $\sigma\left(T_{0}(A)\right)=\sigma(A)$. Indeed, we have

$$
T_{0}(A)=\bigcup_{i=1}^{\infty}\left\{(j, \alpha(i, \tau) \cdot y) \mid(i, y) \in A \text { and } g(i, \tau) \cdot B_{i}=B_{j}\right\} .
$$

Since the map $B_{i} \mapsto g(i, \tau) \cdot B_{i}$ is injective and $\alpha(i, \tau)$ is measure-preserving for all $i$, we have

$$
\begin{aligned}
\sigma\left(T_{0}(A)\right) & =\sum_{i=1}^{\infty} \nu(\{\alpha(i, \tau) \cdot y \mid(i, y) \in A\}) \\
& =\sum_{i=1}^{\infty} \nu(\{y \mid(i, y) \in A\}) \\
& =\sigma(A)
\end{aligned}
$$

Note that by Lemma 2.2 (4), $Z_{0} \subseteq\{0,1, \ldots, n\} \times Y$ for some $n \in \mathbb{N}$ and, in particular, $\sigma\left(Z_{0}\right)<\infty$. This also implies that $T_{0}$ can be extended to a full measure-preserving automorphism $T$ of $Z$. Use Lemma 2.3 (4) to obtain

$$
\begin{aligned}
\int_{Z_{0}} \mu(\Phi(T z) \triangle \tau \cdot \Phi(z)) \mathrm{d} \sigma(z) & \leq \int_{Z_{0}} 3 \epsilon \cdot \mu(\Phi(T z)) \mathrm{d} \sigma(z) \\
& \leq 3 \epsilon \int_{Z} \mu(\Phi(z)) \mathrm{d} \sigma(z) \leq 3 \epsilon
\end{aligned}
$$


Also, by Lemma 2.3 (3) and (2.5),

$$
\begin{aligned}
\int_{Z \backslash Z_{0}} & \mu(\Phi(T z) \triangle \tau \cdot \Phi(z)) \mathrm{d} \sigma(z) \\
\leq & \int_{Z \backslash Z_{0}} \mu(\Phi(T z)) \mathrm{d} \sigma(z)+\int_{Z \backslash Z_{0}} \mu(\tau \cdot \Phi(z)) \mathrm{d} \sigma(z) \\
& =\int_{Z} \mu(\Phi(T z)) \mathrm{d} \sigma(z)-\int_{Z_{0}} \mu\left(\Phi\left(T_{0} z\right)\right) \mathrm{d} \sigma(z)+\int_{Z \backslash Z_{0}} \mu(\Phi(z)) \mathrm{d} \sigma(z) \\
& \leq 1-(1-2 \epsilon) \int_{Z_{0}} \mu(\Phi(z)) \mathrm{d} \sigma(z)+\epsilon \\
\leq & 1-(1-2 \epsilon)(1-\epsilon)+\epsilon \leq 4 \epsilon .
\end{aligned}
$$

Finally, combine (2.7) and (2.8) to obtain

$$
\begin{aligned}
\int_{Z} \mu & (\Phi(T z) \triangle \tau \cdot \Phi(z)) \mathrm{d} \sigma(z) \\
& =\int_{Z_{0}} \mu(\Phi(T z) \triangle \tau \cdot \Phi(z)) \mathrm{d} \sigma(z)+\int_{Z \backslash Z_{0}} \mu(\Phi(T z) \triangle \tau \cdot \Phi(z)) \mathrm{d} \sigma(z) \\
& \leq 7 \epsilon .
\end{aligned}
$$

Now we proceed to construct an invariant state on $B\left(L_{0}^{2}(X)\right)$. For $A \in \operatorname{MALG}(X)$, let

$$
\eta_{A}=\chi_{A}-\mu(A)
$$

where $\chi_{A}$ denotes the characteristic function of the set $A$. Then $\eta_{A} \in L_{0}^{2}(X)$ and $\left\|\eta_{A}\right\|^{2}=\mu(A)-\mu(A)^{2}$. Also, for any $S \in B\left(L_{0}^{2}(X)\right)$,

$$
\begin{aligned}
\left|\left\langle S \eta_{A}, \eta_{A}\right\rangle-\left\langle S \eta_{B}, \eta_{B}\right\rangle\right| & \leq\|S\|\left(\left\|\eta_{A}\right\|+\left\|\eta_{B}\right\|\right)\left\|\eta_{A}-\eta_{B}\right\| \\
& \leq\|S\|(\sqrt{\mu(A)}+\sqrt{\mu(B)}) \sqrt{\mu(A \triangle B)},
\end{aligned}
$$

as is verified by direct computation.

Enumerate $\Gamma=\left\{\gamma_{n}\right\}$ and set $Q_{n}=\left\{\gamma_{1}, \gamma_{2}, \ldots, \gamma_{n}\right\}$. For each $n$, let $n, \Phi_{n}: Z \rightarrow$ MALG $(X)$ be a $\left(Q_{n}, 1 / n\right)$-invariant map as given by Proposition 2.1. Let $M_{n} \in$ $B\left(L_{0}^{2}(X)\right)^{*}$ be the positive linear functional defined by

$$
M_{n}(S)=\int_{Z}\left\langle S \eta_{\Phi_{n}(z)}, \eta_{\Phi_{n}(z)}\right\rangle \mathrm{d} \sigma(z) .
$$

Note that by (ii) and an application of Fubini,

$$
\int_{Z} \mu\left(\Phi_{n}(z)\right) \mathrm{d} \sigma(z)=1 .
$$

Hence,

$$
\begin{aligned}
\left|M_{n}(S)\right| & \leq \int_{Z}\left|\left\langle S \eta_{\Phi_{n}(z)}, \eta_{\Phi_{n}(z)}\right\rangle\right| \mathrm{d} \sigma(z) \\
& \leq \int_{Z}\|S\|\left\|\eta_{\Phi_{n}(z)}\right\|^{2} \mathrm{~d} \sigma(z) \\
& \leq\|S\| \int_{Z} \mu\left(\Phi_{n}(z)\right) \mathrm{d} \sigma(z)=\|S\| .
\end{aligned}
$$


Therefore $\left\|M_{n}\right\| \leq 1$. Now let $M$ be any weak* limit point of the set $\left\{M_{n}\right\}$. We will show that $M$ is a $\kappa_{0}$-invariant state on $B\left(L_{0}^{2}(X)\right)$ which will complete the proof of the theorem. $M$ is clearly positive. Let $I$ denote the identity operator on $L_{0}^{2}(X)$. We have

$$
\begin{aligned}
M_{n}(I) & =\int_{Z}\left\langle\eta_{\Phi_{n}(z)}, \eta_{\Phi_{n}(z)}\right\rangle \mathrm{d} \sigma(z) \\
& =\int_{Z} \mu\left(\Phi_{n}(z)\right)-\mu\left(\Phi_{n}(z)\right)^{2} \mathrm{~d} \sigma(z) \\
& =1-\int_{Z} \mu\left(\Phi_{n}(z)\right)^{2} \mathrm{~d} \sigma(z) \rightarrow 1 \quad \text { as } \quad n \rightarrow \infty .
\end{aligned}
$$

Indeed, by (iii),

$$
\begin{aligned}
\int_{Z} \mu\left(\Phi_{n}(z)\right)^{2} \mathrm{~d} \sigma(z)= & \int_{\left\{\mu\left(\Phi_{n}(z)\right)>1 / n\right\}} \mu\left(\Phi_{n}(z)\right)^{2} \mathrm{~d} \sigma(z) \\
& +\int_{\left\{\mu\left(\Phi_{n}(z)\right) \leq 1 / n\right\}} \mu\left(\Phi_{n}(z)\right)^{2} \mathrm{~d} \sigma(z) \\
\leq & \frac{1}{n}+\frac{1}{n} \int_{Z} \mu\left(\Phi_{n}(z)\right) \mathrm{d} \sigma(z)=\frac{2}{n} .
\end{aligned}
$$

Hence, $M(I)=1$.

To show that $M$ is invariant, it suffices to check that for all $\tau \in \Gamma$ and $S \in$ $B\left(L_{0}^{2}(X)\right)$,

$$
M_{n}\left(\kappa_{0}\left(\tau^{-1}\right) S \kappa_{0}(\tau)\right)-M_{n}(S) \rightarrow 0
$$

Indeed, since $M$ is a weak ${ }^{*}$ limit point of the $M_{n}$ 's, for every $\epsilon>0$ there exist infinitely many $n$ such that

$$
\left|M(S)-M_{n}(S)\right|<\epsilon \quad \text { and } \quad\left|M\left(\kappa_{0}\left(\tau^{-1}\right) S \kappa_{0}(\tau)\right)-M_{n}\left(\kappa_{0}\left(\tau^{-1}\right) S \kappa_{0}(\tau)\right)\right|<\epsilon .
$$

Then

$$
\begin{aligned}
\left|M(S)-M\left(\kappa_{0}\left(\tau^{-1}\right) S \kappa_{0}(\tau)\right)\right| \leq \mid & M(S)-M_{n}(S)|+| M_{n}(S)-M_{n}\left(\kappa_{0}\left(\tau^{-1}\right) S \kappa_{0}(\tau)\right) \mid \\
& +\left|M_{n}\left(\kappa_{0}\left(\tau^{-1}\right) S \kappa_{0}(\tau)\right)-M\left(\kappa_{0}\left(\tau^{-1}\right) S \kappa_{0}(\tau)\right)\right| \\
\leq & \left|M_{n}(S)-M_{n}\left(\kappa_{0}\left(\tau^{-1}\right) S \kappa_{0}(\tau)\right)\right|+2 \epsilon
\end{aligned}
$$

which shows that $M(S)=M\left(\kappa_{0}\left(\tau^{-1}\right) S \kappa_{0}(\tau)\right)$. 
Fix $\tau \in \Gamma$ and $S \in B\left(L_{0}^{2}(X)\right)$. For all $n$ big enough that $\tau \in Q_{n}$, apply Proposition 2.1 to obtain $T_{n} \in \operatorname{Aut}(Z)$, satisfying (iii). Using (2.9) and CauchySchwartz, we have

$$
\begin{aligned}
\left|M_{n}\left(\kappa_{0}\left(\tau^{-1}\right) S \kappa_{0}(\tau)\right)-M_{n}(S)\right| \\
=\left|\int_{Z}\left\langle\kappa_{0}\left(\tau^{-1}\right) S \kappa_{0}(\tau) \eta_{\Phi_{n}(z)}, \eta_{\Phi_{n}(z)}\right\rangle \mathrm{d} \sigma(z)-\int_{Z}\left\langle S \eta_{\Phi_{n}(z)}, \eta_{\Phi_{n}(z)}\right\rangle \mathrm{d} \sigma(z)\right| \\
=\left|\int_{Z}\left\langle S \eta_{\tau \cdot \Phi_{n}(z)}, \eta_{\tau \cdot \Phi_{n}(z)}\right\rangle \mathrm{d} \sigma(z)-\int_{Z}\left\langle S \eta_{\Phi_{n}\left(T_{n} z\right)}, \eta_{\Phi_{n}\left(T_{n} z\right)}\right\rangle \mathrm{d} \sigma(z)\right| \\
\leq \int_{Z}\left|\left\langle S \eta_{\tau \cdot \Phi_{n}(z)}, \eta_{\tau \cdot \Phi_{n}(z)}\right\rangle-\left\langle S \eta_{\Phi_{n}\left(T_{n} z\right)}, \eta_{\Phi_{n}\left(T_{n} z\right)}\right\rangle\right| \mathrm{d} \sigma(z) \\
\leq 2\|S\| \int_{Z}\left(\mu\left(\Phi_{n}(z)\right)^{\frac{1}{2}}+\mu\left(\Phi_{n}\left(T_{n} z\right)\right)^{\frac{1}{2}}\right) \mu\left(\tau \cdot \Phi_{n}(z) \triangle \Phi_{n}\left(T_{n} z\right)\right)^{\frac{1}{2}} \mathrm{~d} \sigma(z) \\
\leq 2\|S\|\left(\left(\int_{Z} \mu\left(\Phi_{n}(z)\right) \mathrm{d} \sigma(z)\right)^{\frac{1}{2}}+\left(\int_{Z} \mu\left(\Phi_{n}\left(T_{n} z\right)\right) \mathrm{d} \sigma(z)\right)^{\frac{1}{2}}\right) \\
\quad \cdot\left(\int_{Z} \mu\left(\tau \cdot \Phi_{n}(z) \triangle \Phi_{n}\left(T_{n} z\right)\right) \mathrm{d} \sigma(z)\right)^{\frac{1}{2}} \\
\quad 2\|S\| \frac{2}{\sqrt{n}} \rightarrow 0 \text { as } n \rightarrow \infty
\end{aligned}
$$

This completes the proof of the theorem.

\section{Amenable Koopman Representations And almost invariant vectors}

In this section, we describe two situations in which the amenability of the Koopman representation is equivalent to the existence of almost invariant vectors $\left(1_{\Gamma} \prec \kappa_{0}\right)$. Note that by (1.2), $1_{\Gamma} \prec \pi$ implies that $\pi$ is amenable for any representation $\pi$, but the converse is not true in general, even for Koopman representations (consider, for example, a modular, ergodic action of a property (T) group). A special situation when it is true is given by the lemma below.

Let $I$ be a countable set and let $\Gamma$ act on $I$. Recall that the action is called amenable if there is a $\Gamma$-invariant mean on $\ell^{\infty}(I)$.

Lemma 3.1. Let $\Gamma$ be a countable group, let $\pi$ be a unitary representation of $\Gamma$ on a separable Hilbert space $\mathcal{H}$ and let $\left\{\xi_{i}\right\}_{i \in \mathbb{N}}$ be an orthonormal basis for $\mathcal{H}$ invariant under $\pi$. Then

$$
\pi \text { is amenable } \Longrightarrow 1_{\Gamma} \prec \pi \text {. }
$$

Proof. Set $I=\left\{\xi_{i}: i \in \mathbb{N}\right\}$. We can identify $\mathcal{H}$ with $\ell^{2}(I)$ and the representation $\pi$ with the representation of $\Gamma$ on $\ell^{2}(I)$ induced by the action of $\Gamma$ on $I$. Let $B(\mathcal{H})$ denote the space of bounded operators on $\mathcal{H} . \pi$ amenable implies that there is a state $M$ on $B(\mathcal{H})$ invariant under $\pi$, i.e.,

$$
M\left(\pi(\gamma) S \pi(\gamma)^{-1}\right)=M(S), \quad \text { for all } \gamma \in \Gamma, S \in B(\mathcal{H})
$$

For each $\phi \in \ell^{\infty}(I)$ consider the multiplication operator $T_{\phi} \in B(\mathcal{H})$ defined by

$$
T_{\phi} f=\phi f
$$

and notice that

$$
\pi(\gamma) T_{\phi} \pi(\gamma)^{-1}=T_{\gamma \cdot \phi}
$$


where $(\gamma \cdot \phi)(\xi)=\phi\left(\pi\left(\gamma^{-1}\right) \cdot \xi\right)$. Hence $\phi \mapsto M\left(T_{\phi}\right)$ defines a $\Gamma$-invariant mean on $\ell^{\infty}(I)$ and the action of $\Gamma$ on $I$ is amenable. But this implies that $1_{\Gamma} \prec \pi$.

Remark. The proof of Lemma 3.1 also shows that if $\Gamma$ acts on a countable set $I$, the corresponding representation is amenable iff the action is amenable.

Now let $\left(X_{0}, \mu_{0}\right)$ be a probability space. If $I$ is countable and $\Gamma \curvearrowright I$, we have a measure-preserving action $\Gamma \curvearrowright X_{0}^{I}$ by permuting the coordinates, which is called a generalized Bernoulli shift.

Proposition 3.2. Let $\Gamma \curvearrowright X_{0}^{I}$ be a generalized Bernoulli shift. Let $\kappa_{0}$ be the corresponding Koopman representation of $\Gamma$. Then the following are equivalent:

(i) the action $\Gamma \curvearrowright I$ is amenable;

(ii) $\kappa_{0}$ is amenable;

(iii) $1_{\Gamma} \prec \kappa_{0}$.

Proof. The equivalence of (ii) and (iii) follows from [18, Theorem 1.2]. From the analysis of the Koopman representation of generalized Bernoulli shifts carried out in [18, Section 3], it follows that there is a basis of $L_{0}^{2}\left(X_{0}^{I}\right)$ invariant under $\kappa_{0}$. Hence Lemma 3.1 applies and we have (iii) $\Rightarrow$ (iii). Lastly, the implication (iii) $\Rightarrow$ (iii) follows from (1.2).

Corollary 3.3. Let $\Gamma \curvearrowright\left(X_{0}, \mu_{0}\right)^{I}$ be a generalized Bernoulli shift. If the action $\Gamma \curvearrowright I$ is non-amenable, then the action $\Gamma \curvearrowright X_{0}^{I}$ is $\mu_{0}^{I}$-antimodular.

Corollary 3.4. Let $\Gamma \curvearrowright\left(X_{0}, \mu_{0}\right)^{I}$ be a mixing generalized Bernoulli shift. Then if $\Gamma$ is non-amenable, the action $\Gamma \curvearrowright X_{0}^{I}$ is $\mu_{0}^{I}$-antimodular.

Proof. $\Gamma \curvearrowright X_{0}^{I}$ mixing implies that for each $i \in I$, the stabilizer $\Gamma_{i}$ is finite (see, e.g., [18, Proposition 2.3]). Let $\lambda_{I}$ be the representation of $\Gamma$ on $\ell^{2}(I)$, let $\lambda_{\Gamma}$ denote the left-regular representation of $\Gamma$, and for $H \leq \Gamma$, let $\lambda_{\Gamma / H}$ be the quasi-regular representation on $\ell^{2}(\Gamma / H)$. Let $A \subseteq I$ be a transversal for the action $\Gamma \curvearrowright I$. Then

$$
\lambda_{I}=\bigoplus_{i \in A} \lambda_{\Gamma / \Gamma_{i}} .
$$

It is not hard to see that $\lambda_{\Gamma / \Gamma_{i}} \leq \lambda_{\Gamma}$ (cf. [18, Lemma 3.3]) and hence, $\lambda_{I} \prec \lambda_{\Gamma}$. By Theorem 1.2 and Proposition 3.2 if the action $\Gamma \curvearrowright X_{0}^{I}$ is not antimodular, $\lambda_{I}$ is amenable. Hence by (1.2),$\lambda_{\Gamma}$ is amenable, contradicting the non-amenability of $\Gamma$.

Hjorth's Theorem 1.1 can now be obtained as a special case of either Corollary 3.3 or Corollary 3.4 if we put $\Gamma=I=\mathbf{F}_{2}, X_{0}=2$ and let $\Gamma$ act on $I$ by left translation.

Now consider the case of an action on a compact Polish group (equipped with its normalized Haar measure) by (topological group) automorphisms.

Proposition 3.5. Let $\Gamma$ act on the compact Polish group $G$ by automorphisms and let $\kappa_{0}$ be the corresponding Koopman representation of $\Gamma$ on $L_{0}^{2}(G)$. Then

$$
\kappa_{0} \text { is amenable } \Longrightarrow 1_{\Gamma} \prec \kappa_{0} .
$$

Proof. Fix an invariant state $M$ on $B\left(L_{0}^{2}(G)\right)$. We adopt the notation from Folland [8. Chapter 5] (see also Kechris 21]). Let $\hat{G}_{0}$ denote the set of (equivalence classes of) non-trivial irreducible representations of $G$. Recall that

$$
\left\{\pi_{i j}: i, j \leq d_{\pi} ; \pi \in \hat{G}_{0}\right\}
$$


is an orthogonal basis for $L_{0}^{2}(G)$, where $d_{\pi}=\operatorname{dim} \pi$ and the $\pi_{i j}$ 's are the matrix coefficients of $\pi$. For $\phi \in \ell^{\infty}\left(\hat{G}_{0}\right)$, define the operator $T_{\phi} \in B\left(L_{0}^{2}(G)\right)$ by

$$
T_{\phi} \pi_{i j}=\phi(\pi) \pi_{i j}
$$

Notice that

$$
T_{\gamma \cdot \phi}=\kappa_{0}(\gamma) T_{\phi} \kappa_{0}(\gamma)^{-1}
$$

and hence $\phi \mapsto M\left(T_{\phi}\right)$ defines a $\Gamma$-invariant mean on $\ell^{\infty}\left(\hat{G}_{0}\right)$.

Let $I=\left\{\chi_{\pi}: \pi \in \hat{G}_{0}\right\}$ (where $\chi_{\pi}$ denotes the character corresponding to $\pi$ ) and notice that $I$ is invariant under $\kappa_{0}$. We can also identify $\ell^{\infty}(I)$ and $\ell^{\infty}\left(\hat{G}_{0}\right)$ and conclude that the action of $\Gamma$ on $I$ is amenable. Denote $Z L_{0}^{2}(G)=\operatorname{span}\left\{\chi_{\pi}\right.$ : $\left.\pi \in \hat{G}_{0}\right\}$ and $\sigma=\left.\kappa_{0}\right|_{Z L_{0}^{2}(G)}$. We can identify $Z L_{0}^{2}(G)$ with $\ell^{2}(I)$, and by the amenability of the action of $\Gamma$ on $I, 1_{\Gamma} \prec \sigma$. But $\sigma \leq \kappa_{0}$, and we are done.

Remark. Note that generalized Bernoulli shifts with a homogeneous base space (i.e., $X_{0}$ non-atomic or purely atomic with atoms of the same measure) are a special case of actions on abelian compact groups by automorphisms. However, for arbitrary $X_{0}$, this is not the case. It is shown in Kechris-Tsankov [18 that for generalized Bernoulli shifts, $1_{\Gamma} \prec \kappa_{0}$ implies the existence of almost invariant sets, while it is open as to whether the same holds for actions on compact Polish groups by automorphisms.

\section{Applications to ORBit equivalence and Borel Reducibility}

Recall that two measure-preserving actions $\Gamma \curvearrowright X$ and $\Delta \curvearrowright Y$ are called orbit equivalent if there exist conull, invariant sets $A \subseteq X$ and $B \subseteq Y$ and a measurable bijection $f: A \rightarrow B$ such that

$$
\forall x, y \in X \quad x E_{\Gamma}^{X} y \Longleftrightarrow f(x) E_{\Delta}^{Y} f(y) .
$$

In this section, we use modular actions and Theorem 1.2 to show that residually finite groups have at least three non-orbit equivalent, free, ergodic actions.

Dye started the theory of orbit equivalence by showing that all ergodic actions of $\mathbb{Z}$ are orbit equivalent. Later, Ornstein and Weiss showed that, in fact, all ergodic actions of amenable groups are orbit equivalent. For all of this, see [17. In the other direction, Schmidt [22] and Connes-Weiss [5] showed that for non-property (T), non-amenable groups have at least two non-orbit equivalent, free, ergodic actions. The invariant they used was $E_{0}$-ergodicity (called strong ergodicity by them) which we proceed to define. $E_{0}$ is the equivalence relation on $2^{\mathbb{N}}$ given by

$$
\left(x_{n}\right) E_{0}\left(y_{n}\right) \Longleftrightarrow \exists n \forall m>n \quad x_{m}=y_{m} .
$$

A measure-preserving group action $\Gamma \curvearrowright X$ (or the orbit equivalence relation it defines) is said to be $E_{0}$-ergodic if for every homomorphism from $E_{\Gamma}^{X}$ to $E_{0}$, there exists a single $E_{0}$ equivalence class whose preimage is conull. For a measurepreserving, ergodic action $\Gamma \curvearrowright X$, not being $E_{0}$-ergodic is equivalent to possessing almost invariant sets, i.e., a sequence of measurable sets $\left\{A_{n}\right\}$ with measures bounded away from 0 and 1 satisfying for each $\gamma \in \Gamma$,

$$
\mu\left(\gamma \cdot A_{n} \triangle A_{n}\right) \rightarrow 0 \quad \text { as } \quad n \rightarrow \infty
$$

(Jones-Schmidt [16]). The definition shows that $E_{0}$-ergodicity is an invariant of orbit equivalence, while the existence of almost invariant sets is usually easier to 
verify in particular cases. For more information on the topic, see Hjorth-Kechris 12 , Appendix A].

Many non-amenable groups are now known to have a continuum of non-orbit equivalent actions: for example, property (T) groups (Hjorth [13]) and non-abelian free groups (Gaboriau-Popa [9]). The latter result was recently extended by Ioana 14 to include all countable groups containing a copy of $\mathbf{F}_{2}$. It is not known whether all non-amenable groups admit a continuum of non-orbit equivalent actions. For more information on orbit equivalence and related topics, see the surveys Gaboriau [10] and Shalom [23] as well as the book Kechris-Miller [17].

First we note the following simple corollary of Theorem 1.2 .

Corollary 4.1. Let $\Gamma \curvearrowright(X, \mu)$ and $\Gamma \curvearrowright(Y, \nu)$ be two measure-preserving actions where the measure $\mu$ is non-atomic. Then if the Koopman representation $\kappa_{0}$ corresponding to the action $\Gamma \curvearrowright X$ is non-amenable, the product action $\Gamma \curvearrowright X \times Y$ is $\mu \times \nu$-antimodular.

Proof. The action $\Gamma \curvearrowright Y$ defines a homomorphism $\phi: \Gamma \rightarrow \operatorname{Aut}(Y)$, and we can take $\alpha(x, \gamma)=\phi(\gamma)$ in Theorem 1.2 .

Theorem 4.2. Suppose that $\Gamma$ is a countable, non-amenable, residually finite group. Then $\Gamma$ has at least three non-orbit equivalent, free, measure-preserving, ergodic actions.

Proof. It is already known that countable groups with property (T) admit continuum many such actions (Hjorth [13]). We thus assume that $\Gamma$ does not have property $(\mathrm{T})$. First we construct two antimodular actions of $\Gamma$ that are not orbit equivalent to each other. Since $\Gamma$ does not have property $(\mathrm{T})$, there is a measurepreserving, ergodic action $\Gamma \curvearrowright(Y, \nu)$ that is not $E_{0}$-ergodic (see Connes-Weiss [5]). Consider the shift action $\Gamma \curvearrowright 2^{\Gamma}$. It is ergodic, free a.e., and $E_{0}$-ergodic (JonesSchmidt [16; cf. Proposition 3.2). By Corollary 3.3, it is also antimodular. Now consider the diagonal action $\Gamma \curvearrowright 2^{\Gamma} \times Y$. It is free a.e. and ergodic. Corollary 4.1 implies that it is also antimodular. Since $\Gamma \curvearrowright Y$ is not $E_{0}$-ergodic, $\Gamma \curvearrowright 2^{\Gamma} \times Y$ is not $E_{0}$-ergodic either (almost invariant sets in $Y$ lift to the product). Finally, since $\Gamma$ is residually finite, there exists a free, modular, ergodic action $\Gamma \curvearrowright Z$.

Now our three actions are $\Gamma \curvearrowright 2^{\Gamma}, \Gamma \curvearrowright 2^{\Gamma} \times Y$, and $\Gamma \curvearrowright Z$. $E_{0}$-ergodicity distinguishes the first two, and by antimodularity, they are not orbit equivalent to the third.

An equivalence relation $E$ on a standard Borel space $X$ is Borel reducible to an equivalence relation $F$ on $Y$ (written as $E \leq_{B} F$ ) if there exists a Borel homomorphism $\pi$ from $E$ to $F$ such that

$$
x E y \Longleftrightarrow \pi(x) F \pi(y) \quad \forall x, y \in X .
$$

$E \leq_{B} F$ expresses that, in some sense, the equivalence relation $F$ is more complicated than $E$. We say that $E$ and $F$ are Borel bireducible if $E \leq_{B} F$ and $F \leq_{B} E$. For more on the subject of Borel reducibility of countable Borel equivalence relations, we refer the reader to Jackson-Kechris-Louveau [15, Hjorth-Kechris [12], and, for motivation and more general background, to Kechris [19].

We have the following application of Theorem 1.2 
Theorem 4.3. Suppose that $\Gamma$ is a countable, non-amenable, residually finite group. Then $\Gamma$ has two free, measure-preserving actions whose orbit equivalence relations are not Borel bireducible.

Proof. In the terminology of the proof of Theorem 4.2 we just consider the equivalence relations $E_{\Gamma}^{2^{\Gamma}}$ and $E_{\Gamma}^{Z}$.

\section{A counterexample}

In this section, we construct an example of a group action orbit equivalent to a modular action (of another group) such that its Koopman representation does not weakly contain any finite-dimensional representation (and, in particular, is weakly mixing).

Let $\Gamma$ be a residually finite group, $\left\{H_{i}\right\}_{i \in I}$ be a countable family of normal subgroups of $\Gamma$ of finite index such that $\bigcap_{i \in I} H_{i}=\{1\}$ and $X=\lim \Gamma / H_{i}$ be the profinite completion of $\Gamma$ with respect to this family. Let $\mu$ be the (normalized) Haar measure on $X$. $\Gamma$ embeds as a dense subgroup of $X$ and the left translation action of $\Gamma$ on $X$ is free, ergodic, and modular (see [21, 5G]). Denote by $\lambda$ the left-regular representation of $X$ on $L^{2}(X)$ and notice that by the density of $\Gamma$ in $X$, a subspace of $L^{2}(X)$ is invariant under $\lambda$ iff it is invariant under $\left.\lambda\right|_{\Gamma}$. Hence, by the Peter-Weyl theorem, any irreducible subrepresentation of $\left.\lambda\right|_{\Gamma}$ is finite-dimensional and has finite multiplicity in $\left.\lambda\right|_{\Gamma}$.

We need the following preliminary lemma.

Lemma 5.1. Let $\pi$ be a finite-dimensional, irreducible representation of a countable group $\Gamma$ with property $(T)$. Then for every normalized positive definite function $\phi$ on $\Gamma$ associated to $\pi$, the following holds: whenever $0 \leq a_{k} \leq 1, \psi_{k}$ and $\theta_{k}$ are normalized positive definite functions on $\Gamma$, the cyclic representations corresponding to the $\theta_{k}$ 's do not contain $\pi$, and $\left(1-a_{k}\right) \psi_{k}+a_{k} \theta_{k} \rightarrow \phi$ pointwise, it is always the case that $a_{k} \rightarrow 0$.

The following proof, simpler than our original one, was suggested by the referee.

Proof. Let $\mathcal{P}_{1}$ be the set of normalized positive definite functions on $\Gamma$ considered as a subset of $\ell^{\infty}(\Gamma)$ equipped with the weak* topology which coincides on $\mathcal{P}_{1}$ with the pointwise convergence topology. Since $\Gamma$ is discrete, $\mathcal{P}_{1}$ is compact. For a positive definite function $\beta$ on $\Gamma$, denote by $\rho_{\beta}$ the cyclic representation associated to $\beta$. We have $\pi=\rho_{\phi}$.

Suppose that $a_{k}$ does not converge to 0 . Then, by the compactness of $\mathcal{P}_{1}$, we can find $\psi, \theta \in \mathcal{P}_{1}$ and $a>0$ such that $\psi_{k_{n}} \rightarrow \psi, \theta_{k_{n}} \rightarrow \theta$ pointwise, and $a_{k_{n}} \rightarrow a$ for some subsequence $\left\{k_{n}\right\} \subseteq \mathbb{N}$. This implies that $(1-a) \psi+a \theta=\phi$. Since $\rho_{\phi}$ is irreducible and $a>0$, we conclude that $\rho_{\theta}=\rho_{\phi}=\pi$ (see [1, C.5.1]). Now, since $\theta_{k_{n}} \rightarrow \theta$ pointwise, it follows that $\rho_{\theta_{k_{n}}} \rightarrow \rho_{\theta}=\pi$ in the Fell topology. Hence $\pi \prec \bigoplus_{n} \rho_{\theta_{k_{n}}}$, and by property (T) and the irreducibility of $\pi$, we conclude that $\pi \leq \rho_{\theta_{k_{n}}}$ for some $n$, a contradiction.

Proposition 5.2. Let $\Gamma$ and $X$ be as in the beginning of the section. If moreover $\Gamma$ has property $(T)$, there exists a group $\Delta$ and an action $\Delta \curvearrowright X$ by measurepreserving transformations generating $E_{\Gamma}^{X}$ such that the Koopman representation of $\Delta$ on $L_{0}^{2}(X)$ does not weakly contain any finite-dimensional representation of $\Delta$ (and, in particular, is weakly mixing). 
Proof. Recall that for a measure-preserving equivalence relation $E$, the full group of $E$ (denoted by $[E]$ ) is the group of all measure-preserving transformations $T$ preserving $E$, i.e., satisfying $T(x) E x$ for almost all $x \in X$. If $T$ is a measurepreserving transformation, $[T]$ denotes the full group of the equivalence relation generated by $T$.

Let $E$ be the equivalence relation induced by the action of $\Gamma$. Since $E$ is ergodic, there is an ergodic $T \in[E]$ (see $[20,3.5]$ ). Hence, $[T] \leq[E]$. Let $\Lambda$ be a non-trivial, countable, amenable group which does not have non-trivial finite-dimensional representations (for example, $\operatorname{SL}\left(2 ; \bar{F}_{2}\right)$, where $\bar{F}_{2}$ is the algebraic closure of the field with two elements; cf. Dye [6]). By Dye's theorem and Ornstein-Weiss (see [17, Theorem 10.7]), we can embed $\Lambda$ in $[T]$ (and therefore in $[E]$ ) so that the resulting action $\Lambda \curvearrowright X$ is ergodic. Let $\Delta$ be the subgroup of $[E]$ generated by $\Gamma$ and $\Lambda$. $\Delta$ inherits a natural action on $X$ from $[E]$. Denote by $\kappa_{0}$ the Koopman representation of $\Delta$ on $L_{0}^{2}(X)$.

Suppose, towards contradiction, that $\pi \prec \kappa_{0}$ for some finite-dimensional representation $\pi$ of $\Delta$ on a Hilbert space $\mathcal{H}_{\pi}$. Without loss of generality, we can assume that $\pi$ is irreducible. By the properties of $\Lambda,\left.\pi\right|_{\Lambda}$ is trivial and hence $\left.\pi\right|_{\Gamma}$ is irreducible. We have $\left.\left.\pi\right|_{\Gamma} \prec \kappa_{0}\right|_{\Gamma}$ and, since $\Gamma$ has property $(\mathrm{T}),\left.\pi\right|_{\Gamma} \leq\left.\kappa_{0}\right|_{\Gamma}$. Let $\mathcal{K}_{\pi}$ be the sum of all subspaces of $L_{0}^{2}(X)$ invariant under $\left.\kappa_{0}\right|_{\Gamma}$ on which $\left.\kappa_{0}\right|_{\Gamma}$ is equivalent to $\left.\pi\right|_{\Gamma}$. Then, by the above observations, $\operatorname{dim} \mathcal{K}_{\pi}<\infty$.

Fix a unit vector $\xi \in \mathcal{H}_{\pi}$. There exists a sequence $\left\{\eta_{k}\right\}$ of unit vectors in $L_{0}^{2}(X)$ such that

$$
\left\langle\kappa_{0}(\delta) \cdot \eta_{k}, \eta_{k}\right\rangle \rightarrow\langle\pi(\delta) \cdot \xi, \xi\rangle \quad \text { for all } \delta \in \Delta .
$$

Set $\phi(g)=\langle\pi(g) \cdot \xi, \xi\rangle$ for $g \in \Gamma$. Write $\eta_{k}=\eta_{k}^{1}+\eta_{k}^{2}$ where $\eta_{k}^{1} \in \mathcal{K}_{\pi}$ and $\eta_{k}^{2} \in \mathcal{K}_{\pi}^{\perp}$, and $\left\|\eta_{k}^{1}\right\|^{2}+\left\|\eta_{k}^{2}\right\|^{2}=1$. Now we have, for all $g \in \Gamma$,

$$
\left\|\eta_{k}^{1}\right\|^{2}\left\langle\kappa_{0}(g) \cdot \frac{\eta_{k}^{1}}{\left\|\eta_{k}^{1}\right\|}, \frac{\eta_{k}^{1}}{\left\|\eta_{k}^{1}\right\|}\right\rangle+\left\|\eta_{k}^{2}\right\|^{2}\left\langle\kappa_{0}(g) \cdot \frac{\eta_{k}^{2}}{\left\|\eta_{k}^{2}\right\|}, \frac{\eta_{k}^{2}}{\left\|\eta_{k}^{2}\right\|}\right\rangle \rightarrow \phi(g),
$$

and Lemma 5.1 allows us to conclude that $\eta_{k}^{2} \rightarrow 0$.

On the other hand, $\left.\kappa_{0}\right|_{\Lambda}$ does not have invariant vectors and hence

$$
\forall 0 \neq \eta \in \mathcal{K}_{\pi} \exists g \in \Lambda \quad\left|\left\langle\kappa_{0}(g) \cdot \eta, \eta\right\rangle\right|<\|\eta\|^{2}
$$

$\left(\left|\left\langle\kappa_{0}(g) \cdot \eta, \eta\right\rangle\right|=\|\eta\|^{2}\right.$ for all $g$ implies that $\left.\kappa_{0}\right|_{\Lambda}$ restricted to $\mathbb{C} \eta$ is a one-dimensional representation of $\Lambda$, hence trivial, and hence $\eta$ is an invariant vector). By compactness (of the unit sphere in $\mathcal{K}_{\pi}$ ), there exists a finite $Q \subseteq \Lambda$ and $\epsilon>0$ such that

$$
\forall 0 \neq \eta \in \mathcal{K}_{\pi} \exists g \in Q \quad\left|\left\langle\kappa_{0}(g) \cdot \eta, \eta\right\rangle\right|<(1-\epsilon)\|\eta\|^{2} .
$$

Let $k$ be so large that $\left\|\eta_{k}^{2}\right\|<\epsilon / 4$. Then we calculate, for any $g \in \Lambda$,

$$
\begin{aligned}
\left|\left\langle\kappa_{0}(g) \cdot \eta_{k}, \eta_{k}\right\rangle\right| & =\left|\left\langle\kappa_{0}(g) \cdot\left(\eta_{k}^{1}+\eta_{k}^{2}\right), \eta_{k}^{1}+\eta_{k}^{2}\right\rangle\right| \\
& \leq\left|\left\langle\kappa_{0}(g) \cdot \eta_{k}^{1}, \eta_{k}^{1}\right\rangle\right|+\left|\left\langle\kappa_{0}(g) \cdot \eta_{k}^{2}, \eta_{k}^{2}\right\rangle\right|+2\left\|\eta_{k}^{1}\right\|\left\|\eta_{k}^{2}\right\| \\
& \leq\left|\left\langle\kappa_{0}(g) \cdot \eta_{k}^{1}, \eta_{k}^{1}\right\rangle\right|+\left\|\eta_{k}^{2}\right\|^{2}+2\left\|\eta_{k}^{1}\right\|\left\|\eta_{k}^{2}\right\| \\
& \leq\left|\left\langle\kappa_{0}(g) \cdot \eta_{k}^{1}, \eta_{k}^{1}\right\rangle\right|+3 \epsilon / 4 .
\end{aligned}
$$

But by (5.1), for each $\eta_{k}^{1}$, there exists $g \in Q$ such that $\left|\left\langle\kappa_{0}(g) \cdot \eta_{k}^{1}, \eta_{k}^{1}\right\rangle\right|<1-\epsilon$. Therefore there exists $g_{0} \in Q$ such that for infinitely many $k$ 's,

$$
\left|\left\langle\kappa_{0}\left(g_{0}\right) \cdot \eta_{k}, \eta_{k}\right\rangle\right|<1-\epsilon / 4
$$


and, in particular,

$$
\left\langle\kappa_{0}\left(g_{0}\right) \cdot \eta_{k}, \eta_{k}\right\rangle \nrightarrow 1=\left\langle\pi\left(g_{0}\right) \cdot \xi, \xi\right\rangle,
$$

a contradiction.

\section{ACKNOWLEDGEMENTS}

The authors would like to thank their respective advisors G. Hjorth and A. S. Kechris for encouragement, support, and valuable discussions on the topic of this paper. The authors are also grateful to the anonymous referee for suggesting a simplified proof of Lemma 5.1.

\section{REFERENCES}

[1] B. Bekka, P. de la Harpe, and A. Valette, Kazhdan's property (T), available at http://www.mmas.univ-metz.fr/ 'bekka/.

[2] M. E. B. Bekka and A. Valette, Kazhdan's property (T) and amenable representations, Math. Z. 212 (1993), no. 2, 293-299. MR.1202813 (94a:22006)

[3] M. E. B. Bekka, Amenable unitary representations of locally compact groups, Invent. Math. 100 (1990), no. 2, 383-401. MR.1047140 (91g:22007)

[4] P.-A. Cherix, M. Cowling, P. Jolissaint, P. Julg, and A. Valette, Groups with the Haagerup property, Progress in Mathematics, vol. 197, Birkhäuser Verlag, Basel, 2001. MR1852148 (2002h:22007)

[5] A. Connes and B. Weiss, Property T and asymptotically invariant sequences, Israel J. Math. 37 (1980), no. 3, 209-210. MR599455 (82e:28023b)

[6] H. A. Dye, On the ergodic mixing theorem, Trans. Amer. Math. Soc. 118 (1965), 123-130. MR0174705 (30:4905)

[7] J. Feldman and C. C. Moore, Ergodic equivalence relations, cohomology, and von Neumann algebras. I, Trans. Amer. Math. Soc. 234 (1977), no. 2, 289-324. MR0578656 (58:28261a)

[8] G. B. Folland, A course in abstract harmonic analysis, Studies in Advanced Mathematics, CRC Press, Boca Raton, FL, 1995. MR1397028 (98c:43001)

[9] D. Gaboriau and S. Popa, An uncountable family of nonorbit equivalent actions of $\mathbb{F}_{n}$, J. Amer. Math. Soc. 18 (2005), no. 3, 547-559 (electronic). MR2138136

[10] D. Gaboriau, On orbit equivalence of measure preserving actions, Rigidity in dynamics and geometry (Cambridge, 2000), 2002, pp. 167-186. MR1919400(2003c:22027)

[11] E. Glasner, Ergodic theory via joinings, Mathematical Surveys and Monographs, vol. 101, American Mathematical Society, Providence, RI, 2003. MR 1958753 (2004c:37011)

[12] G. Hjorth and A. S. Kechris, Rigidity theorems for actions of product groups and countable Borel equivalence relations, Mem. Amer. Math. Soc. 177 (2005), no. 833, viii+109. MR.2155451 (2006f:03078)

[13] G. Hjorth, A converse to Dye's theorem, Trans. Amer. Math. Soc. 357 (2005), no. 8, 30833103 (electronic). MR2135736 (2005m:03093)

[14] A. Ioana, Orbit inequivalent actions for groups containing a copy of $\mathbb{F}_{2}$ (2007, preprint), available at arXiv:math.GR/0701027.

[15] S. Jackson, A. S. Kechris, and A. Louveau, Countable Borel equivalence relations, J. Math. Log. 2 (2002), no. 1, 1-80. MR1900547 (2003f:03066)

[16] V. F. R. Jones and K. Schmidt, Asymptotically invariant sequences and approximate finiteness, Amer. J. Math. 109 (1987), no. 1, 91-114. MR878200 (88h:28021)

[17] A. S. Kechris and B. D. Miller, Topics in orbit equivalence, Lecture Notes in Mathematics, vol. 1852, Springer-Verlag, Berlin, 2004. MR2095154 (2005f:37010)

[18] A. S. Kechris and T. Tsankov, Amenable actions and almost invariant sets, Proc. Amer. Math. Soc. 136 (2008), no. 2, 687-697. MR2358510(2009a:28035)

[19] A. S. Kechris, New directions in descriptive set theory, Bull. Symbolic Logic 5 (1999), no. 2, 161-174. MR.1791302 (2001h:03090)

[20] - Global aspects of ergodic group actions and equivalence relations (2005 preprint).

[21] _ Unitary representations and modular actions, J. Math. Sci. (New York) 140 (2007), no. $3,398-425$. 
[22] K. Schmidt, Asymptotically invariant sequences and an action of $\mathrm{SL}(2, \mathbf{Z})$ on the 2-sphere, Israel J. Math. 37 (1980), no. 3, 193-208. MR599454(82e:28023a)

[23] Y. Shalom, Measurable group theory, European Congress of Mathematics, 2005, pp. 391-423. MR2185757 (2006k:37007)

[24] S. Thomas, Popa superrigidity and countable Borel equivalence relations (2006, preprint).

Department of Mathematics, University of California, Mathematical Sciences Building 6363, Los Angeles, California 90095

E-mail address: iepstein@math.ucla.edu

Department of Mathematics 253-37, California Institute of Technology, Pasadena, CaLifornia 91125

E-mail address: todor@caltech.edu 